Urologe 2021 · 60:289-290

https://doi.org/10.1007/s00120-021-01460-5

Angenommen: 15. Januar 2021

Online publiziert: 9. Februar 2021

(c) Springer Medizin Verlag GmbH, ein Teil von Springer Nature 2021

\author{
T. Klotz' $\cdot$ M. S. Michel ${ }^{2}$ \\ ' Klinik für Urologie, Andrologie und Kinderurologie, Kliniken Nordoberpfalz AG, Weiden, Deutschland \\ ${ }^{2}$ Universitätsklinik für Urologie und Urochirurgie, Universitätsmedizin Mannheim, Universität Heidelberg, \\ Mannheim, Deutschland
}

\title{
Zwei Runden gehen an Corona - aber die Urologie wird am Ende gewinnen!
}

Die Corona-Pandemie bestimmt das medizinische und gesellschaftliche Leben in den letzten Monaten, und ein Ende ist aktuell noch nicht abzusehen. Während in der ersten Welle im März 2020 die operative urologische Versorgung, vom BMG angeordnet, vielerorts auf dringliche Eingriffe heruntergefahren wurde und Betten und OP-Säle leer standen, zeigte sich, dass im Ergebnis tatsächlich nur einige Hotspotregionen durch COVID-Patienten in den Kliniken belastet waren. In der zweiten Welle ist die gesamte medizinische Standardversorgung beeinträchtigt. Die urologische Notfallversorgung findet statt, aber intensivpflichtige Eingriffe oder größere Interventionen müssen in den meisten Kliniken täglich mit den Koordinatoren vor Ort individuell besprochen werden.

Welche Auswirkungen die Verschiebung von größeren uroonkologischen Eingriffen hat, wissen wir nicht wirklich. Genauso wenig kann eingeschätzt werden, ob es eine Abnahme der Präventionsbereitschaft oder eine relevante Symptomverschleppung aufgrund von Ansteckungsängsten gibt.

Zudem belastet die extrem angespannte Personalsituation in den Kliniken und Praxen aufgrund von Infektionen und Quarantäne. Eine Art von Depression und Fatalismus ist häufig spürbar. Nicht vergessen werden darf die Mehrbelastung von Mitarbeiter/ innen mit schulpflichtigen Kindern in der Lockdown-Situation. Einzelne Beiträge in diesem Heft greifen die Situation in Teilen analytisch auf.
Ein weiterer unfairer Tiefschlag, dessen Bedeutung in der Corona-Pandemie fast untergegangen ist, war die Ablehnung des PSA-Screenings als GKV-Leistung durch den gemeinsamen Bundesausschuss (GBa). Unfair deshalb, weil diese Ablehnung entgegen der ausdrücklichen Empfehlung unserer wissenschaftlichen Fachgesellschaft und entgegen einer breiten wissenschaftlichen Evidenz zu Gunsten eines risikoadaptierten Einsatzes des PSA-Tests erfolgte. Auf der einen Seite Evidenz energisch fordern und sich dann entgegen der Evidenz entscheiden dieses Handeln bestimmt nicht zum ersten Mal die Gesundheitspolitik. Es wäre eine Unterstellung zu denken, dass die vermeintliche zusätzliche ökonomische Belastung des Gesundheitswesens in Post-Pandemiezeiten ein Grund für die Ablehnung sein könnte. Dies wäre ja auch zu kurzfristig gedacht und in den Auswirkungen gerade von Seiten einer ökonomischen Betrachtungsweise peinlich kurzsichtig.

Momentan, in Zeiten der Coronavirus-Pandemie, steht die Urologie nicht primär im Fokus der medizinischen Aufmerksamkeit, wenn man von der Basis- und Notfallversorgung absieht. Aber für uns spricht die Zeit!

Die Impfung wird hoffentlich im Laufe des Jahres 2021 epidemiologisch wirksam - und selbst wenn die Virulenz des Virus in seinen Mutationen aktuell höher zu sein scheint, zeigt sich eine eher geringere Morbidität bei besseren spezifischen 
Therapiemöglichkeiten. Auch hierzu finden Sie einen Beitrag in diesem Heft.

Patienten mit urologischen Problemen werden trotz Pandemie mehr, und dies gilt für alle Bereiche unseres Fachs. Vielleicht haben wir sogar das Glück, dass im Nachgang der Pandemie endlich das DRG-System reformiert wird und die Unternehmensberatungen sich mit ihren Laptops aus den Kliniken zurückziehen.

Wir sind zuversichtlich für die Urologie als Fach und für alle unsere Mitarbeiter/innen, denn unsere Patienten brauchen uns. Wir bieten hochmoderne engagierte Medizin nahe am Menschen und haben im medizinischen Fächerkanon seit Jahren mit die höchste Innovationsdichte. Ein Schwergewichtskampf geht über 12 Runden - die Urologie und damit wir alle, werden gewinnen!

\section{T. Klotz und M.S. Michel}

\section{Korrespondenzadresse}

Prof. Dr. T. Klotz, MPH
Klinik für Urologie, Andrologie
und Kinderurologie, Kliniken
Nordoberpfalz AG
Söllnerstr. 16,92637 Weiden,
Deutschland
theodor.klotz@
kliniken-nordoberpfalz.ag

Interessenkonflikt. T. Klotz und M. S. Michel geben an, dass kein Interessenkonflikt besteht.

\section{Neuerscheinungen in der Urologie}

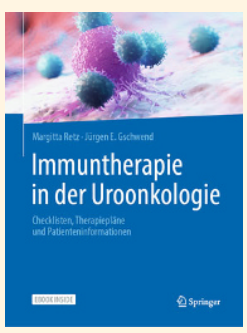

Immuntherapie in der Uroonkologie

Retz, M., Gschwend, J. E.

1.Auflage, 365 Seiten, inkl. Ebook, 79,99EUR, ISBN 978-3-662-60977-4

Vereinfacht dem Arzt die onkologische Therapie durch klare Handlungsanweisungen in Form von Checklisten, Patienteninformationsblätter und Therapieplänen

In einzigartiger Form bündelt das Buch das aktuelle Wissen, welches der Arzt für die sichere Behandlung zur Versorgung von urologischen Tumorpatienten benötigt. Zahlreiche Checklisten,

Tabellen, Therapiepläne und Patienteninformationen machen ein schnelles Nachschlagen möglich. Erstmalig wurde das Nebenwirkungsmanagement von immunvermittelten Toxizitäten auf der Grundlage von internationalen Leitlinien in Tabellenform aufgelistet. Weitere Schwerpunkte sind die Pharmakologie von Immun- und Targettherapeutika unter besonderer Berücksichtigung der Nebenwirkungsprofile und Kontraindikationen.

Die übersichtlich gestalteten Therapieschemata und Patienteninformationen können als Vorlage für die eigene Arbeit genutzt werden - sie sind druckbar und kopierbar. Für den direkten Einsatz während der Therapie erhalten Sie zusätzlich das eBook.

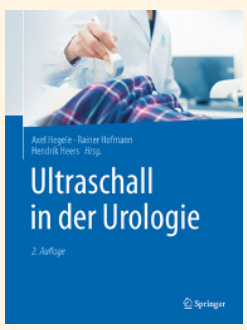

Ultraschall in der Urologie

Hegele, A., Hofmann, R., Heers, $H$.

2.Auf., 339 Seiten, 129,99EUR (April 2021) ISBN 978-3-662-60396-3

Der vorliegende Atlas ist Lehrbuch und Nachschlagewerk zugleich. Er liefert dem klinisch tätigen Urologen einen reichen Fundus von über 400 Referenzaufnahmen häufiger Befunde und interessanter Raritäten. Neben technischen Grundlagen der Sonographie sowie praktischen Tipps zur Vermeidung von Fehlbefunden finden Sie umfangreiche Informationen zur sonographischen Diagnostik benigner und maligner urologischer Erkrankungen. Auf Normbefunde und Normvarianten wird zum besseren Verständnis gezielt eingegangen. Aktuelle Forschungsergebnisse und differenzialdiagnostische Tabellen zur schnellen Kontrolle der gestellten Diagnose runden das Werk ab. Die Auswahl der Bilder folgte höchsten Qualitätsansprüchen. Komplexe Befunde werden zusätzlich durch aussagekräftige Graphiken veranschaulicht.

"Ultraschall in der Urologie", ein unverzichtbarer Begleiter für alle, die sich mit der sonographischen Diagnostik des Urogenitaltrakts befassen.

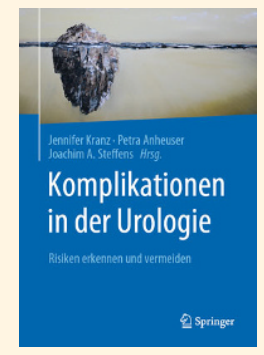

\section{Komplikationen in der Urologie}

Kranz, J., Anheuser, P., Steffens, J. A. 1.Aufl., 478 Seiten, 79,99 EUR (April 2021) ISBN 978-3-662-60624-7 Systematische Darstellung von Komplikationen in der diagnostischen, konservativen und operativen Urologie

Dieses Buch schildert Komplikationen und Zwischenfälle aus dem gesamten Fachbereich der Urologie. Neben konservativen und operativen Therapieoptionen werden auch die Indikationsstellung verschiedener Verfahren sowie diagnostische Maßnahmen einbezogen.

Unter Berücksichtigung des aktuellen Wissenstandes bietet das Buch Strategien zur Vermeidung und Therapievorschläge zur Beherrschung von Komplikationen. Häufigkeitsangaben typischer, aber auch seltener Komplikationen werden systematisch aufgeführt. Der urologisch tätige Arzt erhält Tipps für ein strukturiertes Risikomanagement.

Für alle operativ tätigen Urologen und niedergelassenen Fachärzte, aber auch zur Erstellung von Gutachten, dient es als wertvolle Lektüre. 